\title{
Algorithms Meet Art, Puzzles, and Magic
}

\author{
Erik D. Demaine \\ MIT Computer Science and Artificial Intelligence Laboratory \\ 32 Vassar Street, Cambridge, MA 02139, USA \\ edemaine@mit.edu
}

\begin{abstract}
When I was six years old, my father Martin Demaine and I designed and made puzzles as the Erik and Dad Puzzle Company, which distributed to toy stores across Canada. So began our journey into the interactions between algorithms and the arts. More and more, we find that our mathematical research and artistic projects converge, with the artistic side inspiring the mathematical side and vice versa. Mathematics itself is an art form, and through other media such as sculpture, puzzles, and magic, the beauty of mathematics can be brought to a wider audience. These artistic endeavors also provide us with deeper insights into the underlying mathematics, by providing physical realizations of objects under consideration, by pointing to interesting special cases and directions to explore, and by suggesting new problems to solve (such as the metapuzzle of how to solve a puzzle). This talk will give several examples in each category, from how our first font design led to a universality result in hinged dissections, to how studying curved creases in origami led to sculptures at MoMA. The audience will be expected to participate in some live magic demonstrations.
\end{abstract}

